FOOD \& FUNCTION

\title{
Lemon balm extract causes potent antihyperglycemic and antihyperlipidemic effects in insulin-resistant obese mice
}

\author{
Christopher Weidner ${ }^{1,2}$, Sylvia J. Wowro ${ }^{1}$, Anja Freiwald ${ }^{1}$, Vitam Kodelja ${ }^{1}$, Heba Abdel-Aziz ${ }^{3}$, \\ Olaf Kelber ${ }^{3}$ and Sascha Sauer ${ }^{1}$
}

${ }^{1}$ Otto Warburg Laboratory, Max Planck Institute for Molecular Genetics, Berlin, Germany

${ }^{2}$ Department of Biology, Chemistry, and Pharmacy, Free University of Berlin, Berlin, Germany

${ }^{3}$ Scientific Department, Steigerwald Arzneimittelwerk GmbH, Darmstadt, Germany

Over the last decades polyetiological metabolic diseases such as obesity and type 2 diabetes have emerged as a global epidemic. Efficient strategies for prevention and treatment include dietary intervention and the development of validated nutraceuticals. Safe extracts of edible plants provide a resource of structurally diverse molecules that can effectively interfere with multifactorial diseases. In this study, we describe the application of ethanolic lemon balm (Melissa officinalis) leaves extract for the treatment of insulin-resistance and dyslipidemia in mice. We show that lemon balm extract (LBE) activates the peroxisome proliferator-activated receptors (PPARs), which have key roles in the regulation of whole body glucose and lipid metabolism. Application of LBE $(0.6 \mathrm{mg} / \mathrm{mL})$ to human primary adipocytes resulted in specific peroxisome proliferator-activated receptor target gene expression. LBE treatment of insulin-resistant highfat diet-fed C57BL/6 mice ( $200 \mathrm{mg} / \mathrm{kg} /$ day) for 6 weeks considerably reduced hyperglycemia and insulin resistance, plasma triacylglycerol, nonesterified fatty acids and LDL/VLDL cholesterol levels. Taken together, ethanolic lemon balm extract can potentially be used to prevent or concomitantly treat type 2 diabetes and associated disorders such as dyslipidemia and hypercholesterolemia.

\section{Keywords: \\ Diabetes / Dyslipidemia / Functional Food / Melissa officinalis / Peroxisome proliferator-activated receptor}

Additional supporting information may be found in the online version of this article at the publisher's web-site

Metabolic diseases such as type II diabetes are the result of life style changes over the last decades [1], which require tailored approaches for more efficient intervention. A key component of such endeavours consists of the development of dietary interventions including complementation with functional food or nutraceuticals to combat metabolic disorders.

Correspondence: Dr. Sascha Sauer, Otto Warburg Laboratory, Max Planck Institute for Molecular Genetics, Ihnestrasse 63-73, 14195 Berlin, Germany

E-mail: sauer@molgen.mpg.de

Abbreviations: BFB, bezafibrate; FABP4, fatty acid binding protein 4; HFD, high-fat diet; LBE, lemon balm extract; PDK4, pyruvate dehydrogenase kinase 4; PPAR, peroxisome proliferator-activated receptor; $\mathbf{R G Z , ~ r o s i g l i t a z o n e ~}$
Received: July 1, 2013

Revised: September 2, 2013 Accepted: September 17, 2013
Peroxisome proliferator-activated receptors (PPARs) belong to the class of nuclear receptors and regulate transcriptional activity that can be modulated by binding of a large variety of small ligands such as fatty acids [2]. These nuclear receptors play crucial roles as molecular sensors of environmental factors including nutritional constituents to finetune glucose and lipid homeostasis, and are also important for other physiological processes [2]. Three subtypes of PPARs have been described, namely PPAR- $\alpha$, PPAR- $\beta / \delta$, and PPAR$\gamma$, which are expressed in many cell types, including metabolic target cells such as adipocytes [3]. Depending on the context, the PPARs can (in part) complement the various actions of each other. Specific activation of PPAR- $\alpha$ or PPAR- $\beta / \delta$ by small molecules counteracts various metabolic diseases including dyslipidemia [4]. Synthetic agonists of PPAR- $\gamma$ such as the thiazolidinediones are efficient antidiabetic molecules 


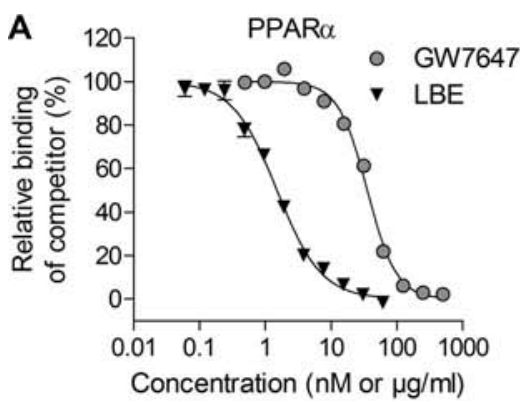

D

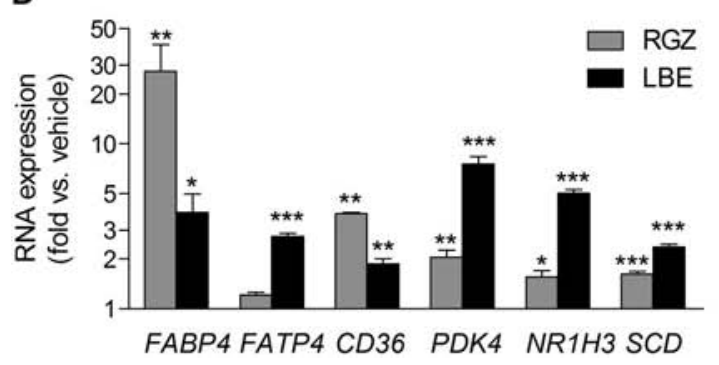

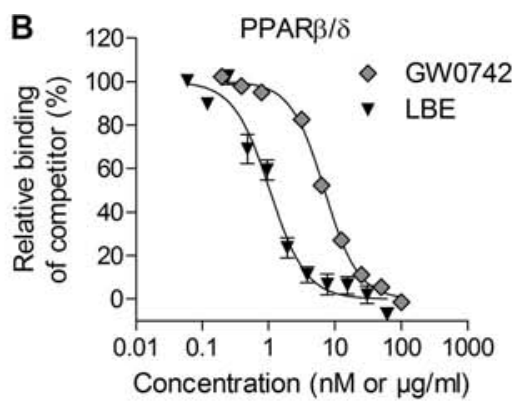
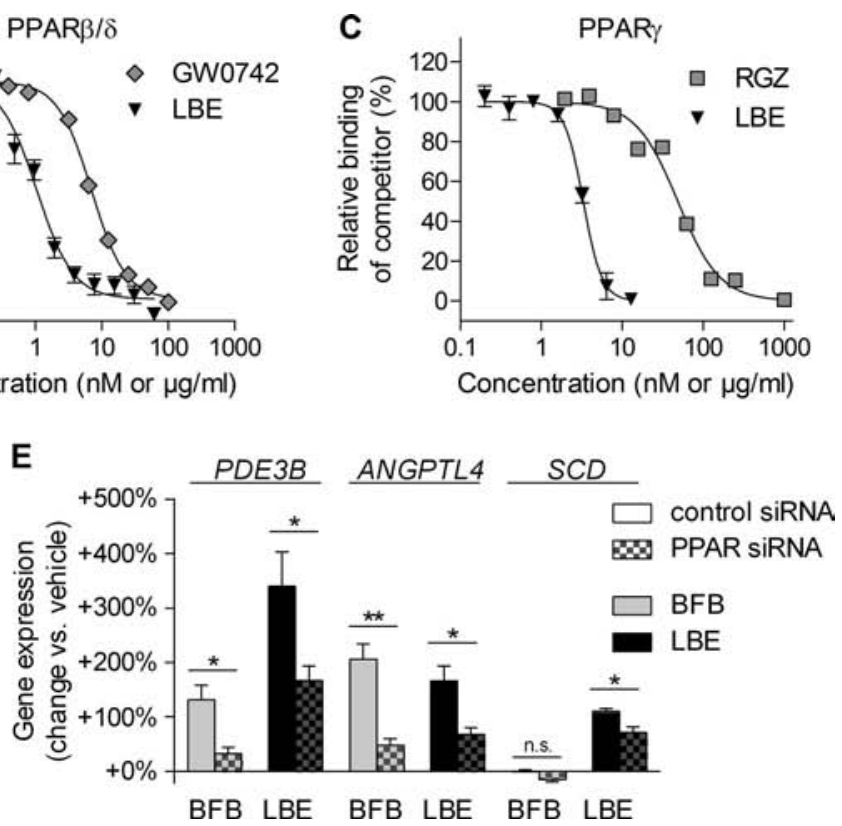

Figure 1. In vitro and cell culture studies with lemon balm extract. (A-C) Binding to PPAR $\alpha$, PPAR $\beta / \delta$ and PPAR $\gamma$ by natural-products contained in lemon balm extract $(\mathrm{LBE}, \mu \mathrm{g} / \mathrm{ml})$ or reference ligands $(\mathrm{nM})$. Binding of compounds was measured in a competitive timeresolved fluorescence resonance energy transfer assay. Data are expressed as mean $\pm S D(n=3)$. (D) Gene expression profile of lemon balm extract (LBE) and rosiglitazone (RGZ) in human adipocytes. Primary adipocytes were treated with either $10 \mu \mathrm{M}$ RGZ, $0.6 \mathrm{mg} / \mathrm{ml} \mathrm{LBE}$ or vehicle only for $24 \mathrm{~h}$ and gene expression was analyzed with qPCR. (E) Human primary adipocytes were transfected with siRNA against PPAR $\alpha, P P A R \beta / \delta$ and PPAR $\gamma$ (checked bars) or control siRNA (solid bars) and were treated with either $300 \mu M$ of the PPAR pan-agonist bezafibrate (BFB), $0.2 \mathrm{mg} / \mathrm{ml}$ LBE or vehicle only for $24 \mathrm{~h}$. Data are expressed as mean $\pm \mathrm{SEM}$ ( $\mathrm{n}=3-4 / \mathrm{group}) .{ }^{*} p \leq 0.05 ;{ }^{* *} p \leq 0.01 ;{ }^{* * *} p$ $\leq 0.001$, vs. vehicle.

but showed a number of unwanted side effects including weight gain, hepatotoxicity, edema, heart failure, osteoporosis, and cancer [5]. Activation of all three PPAR subtypes by pan-agonists has been proposed to combine the antihyperglycemic and insulin-sensitising effects of PPAR- $\gamma$ agonism with the beneficial properties of PPAR- $\alpha$ agonism, that is, the lowering of triacylglycerols and the increase of highdensity lipoprotein cholesterol levels [6]. But unfortunately, many of these synthetic molecules including the dual PPAR$\alpha / \gamma$-activating glitazares were associated with adverse side effects [7].

In general, the number of approved new chemical entities has been declining for the past decade. Consequently, the dominant single-component/single-target concept of pharmaceutical development may hit the limits for the efficient treatment of complex disorders. Multicomponent and/or preventive strategies and the application of mixtures of natural products might overcome current limitations and improve the efficacy/toxicity balance [8].

Plants provide a natural resource for treating diverse complex disorders [9]. Extracts from edible biomaterials have become a major focus of nutritional research to develop functional food and nutraceuticals with proven health benefits and good safety profiles [10]. Here, we report on the benefial effects of a safe ethanolic extract of lemon balm (Melissa officinalis) leaves on treating hyperglycemia and dyslipidemia in insulin-resistant high-fat diet-fed mice (where HFD is, highfat diet).

Initially, we analyzed binding of PPARs by the ingredients of a standardized hydroethanolic lemon balm extract (LBE) $[11,12]$ using a competitive binding assay. Titration of the PPAR ligand-binding domain with LBE revealed an overall binding affinity constant of $0.6,0.4$, and $1.2 \mu \mathrm{g} / \mathrm{mL}$ for PPAR- $\alpha,-\beta / \delta$, and $-\gamma$, respectively (Fig. $1 \mathrm{~A}-\mathrm{C}$ and Table 1 ). In transcriptional reporter gene assays, LBE showed effective concentrations $\left(\mathrm{EC}_{50}\right.$ ) of 46,882 , and $82 \mu \mathrm{g} / \mathrm{mL}$ for PPAR- $\alpha$, $-\beta / \delta$, and $-\gamma$, respectively, with a maximal potency of 50,32 , and $12 \%$ compared to known full PPAR agonists (Supporting Information Fig. 1 and Table 1). These results suggest that LBE comprises pan-PPAR agonists that may regulate PPARdirected gene expression in metabolically relevant target cells.

We therefore tested the ability of LBE to induce PPARregulated gene expression in primary human adipocytes. LBE-treated cells showed increased expression of exemplary target genes of the three PPAR subtypes such as the fatty acid binding protein 4 (FABP4), the fatty acid transport protein 4 , the CD36 molecule, the pyruvate dehydrogenase kinase 4 (PDK4), the liver X receptor alpha (LXR- $\alpha$ or NR1H3), or the lipogenic stearoyl-CoA desaturase (Fig. 1D). FABP4 expression is a marker of adipogenesis and lipid accumulation [13], and fatty acid transport protein 4 as well as CD36 mediate fatty acid transport into the adipocytes. PDK4 regulates 
Table 1. PPAR binding and activation data of LBE and reference compounds

\begin{tabular}{|c|c|c|c|c|}
\hline & LBE & GW7647 & GW0742 & RGZ \\
\hline \multicolumn{5}{|l|}{ PPAR- $\alpha$} \\
\hline $\mathrm{K}_{\mathrm{i}}(\mu \mathrm{g} / \mathrm{mL}$ or $\mathrm{nM})$ & 0.6 & 13 & n.d. & n.d. \\
\hline $\mathrm{EC}_{50}(\mu \mathrm{g} / \mathrm{mL}$ or $\mathrm{nM})$ & 46 & 0.2 & n.d. & n.d. \\
\hline Efficacy (\%) & 50 & 100 & n.d. & n.d. \\
\hline \multicolumn{5}{|l|}{ PPAR- $\beta / \delta$} \\
\hline $\mathrm{K}_{\mathrm{i}}(\mu \mathrm{g} / \mathrm{mL}$ or $\mathrm{nM})$ & 0.4 & n.d. & 3 & n.d. \\
\hline $\mathrm{EC}_{50}(\mu \mathrm{g} / \mathrm{mL}$ or $\mathrm{nM})$ & 882 & n.d. & 0.2 & n.d. \\
\hline Efficacy (\%) & 32 & n.d. & 100 & n.d. \\
\hline \multicolumn{5}{|l|}{ PPAR- $\gamma$} \\
\hline $\mathrm{K}_{\mathrm{i}}(\mu \mathrm{g} / \mathrm{mL}$ or $\mathrm{nM})$ & 1.2 & n.d. & n.d. & 17 \\
\hline $\mathrm{EC}_{50}(\mu \mathrm{g} / \mathrm{mL}$ or $\mathrm{nM})$ & 82 & n.d. & n.d. & 4 \\
\hline Efficacy (\%) & 12 & n.d. & n.d. & 100 \\
\hline
\end{tabular}

LBE data are presented in $\mu \mathrm{g} / \mathrm{mL}, \mathrm{GW} 7647$, GW0742, and RGZ data are given in $n M$. LBE, lemon balm extract $(\mu \mathrm{g} / \mathrm{mL})$; $R G Z$, rosiglitazone $(\mathrm{nM})$; n.d. not determined.

the switch between glucose and fatty acid metabolism, and increased PDK4 expression indicates intensified entry of fatty acid oxidation-derived acetyl-CoA into the tricarboxylic acid cycle. LXR- $\alpha$ is a nuclear transcription factor that has important roles in the regulation of antiinflammatory gene expression. Of note, the increase in FABP4 expression was sevenfold lower with LBE than with the anti-diabetes drug and PPAR- $\gamma$ agonist rosiglitazone (RGZ) (Fig. 1D), indicating lower induction of adipocyte differentiation a process that might be associated with increased adiposity and body weight gain in vivo. Knockdown of all three PPAR subtypes in these cells by small interfering RNA mediated RNA interference (Supporting Information Fig. 2) led to significant reduction of the LBE-induced expression of PPAR target genes, including the cGMP-inhibited phosphodiesterase 3B, the angiopoietin-like 4 and the stearoyl-CoA desaturase gene (Fig. 1E). These results indicate LBE-driven modulation of PPAR activity in primary human adipocytes. Residual gene expression and physiological activity may have been maintained by low amounts of PPARs that can synergistically complement the function of each other, other potentially compensating nuclear receptors that could also interact with the ingredients of LBE such as the LXRs [14,15], or other off-targets.

We then investigated the effects of LBE in an established mouse model of mild insulin resistance [16]. We first induced insulin resistance and dyslipidemia in C57BL/ 6 mice by feeding a HFD for 18 weeks, and then treated these obese mice for 6 weeks with $200 \mathrm{mg} / \mathrm{kg} /$ day LBE, $4 \mathrm{mg} / \mathrm{kg} /$ day RGZ or vehicle via drinking water. LBE-treatment had no significant effect on food intake or body weight gain compared to untreated mice (Supporting Information Fig. 3). After 2 weeks of treatment LBE-treated mice revealed significantly reduced concentrations of fasting blood glucose $(14 \%$ decrease versus vehicle, $p=0.001$ ) equally to the potent antidiabetic drug RGZ (Fig. 2A), whereas LBE showed no significant effects on fasting plasma insulin levels (Fig. 2B). Combining both parameters by application of the homeostatis model assessment of insulin resistance, LBE significantly decreased the HFD-induced insulin resistance by $35 \%(p=0.03$, Fig. 2 C), approximately half as potent than RGZ $(71 \%$ decrease, Fig. 2C). These results indicate strong antidiabetic effects of LBEs in HFD-fed mice.

Moreover, LBE efficiently reduced the HFD-provoked rise in fasting plasma concentrations of nonesterified fatty acids by $59 \%(p=0.0006$, Fig. $2 \mathrm{D})$ and further potently decreased the plasma triacylglycerol gain by $66 \%(p=0.00006$, Fig. 2E), even better than RGZ. Strikingly, in contrast to RGZ, which selectively activates the PPAR- $\gamma$ subtype, LBE-fed mice showed reduced plasma levels of LDL/VLDL cholesterol (32\% decrease, $p=0.006$, Fig. $2 \mathrm{~F}$ ), and a slight decrease in total plasma cholesterol ( $8 \%$ decrease, $p=0.2$, Fig. $2 \mathrm{~F}$ ). LBE treatment led to an increase of the HDL/LDL ratio of $56 \%$ $(p=0.0008$; Fig. 2F), whereas RGZ showed no effect. These data suggest that HFD-induced dyslipidemia can potently be improved with LBE.

Lemon balm (M. officinalis) is a popular herb of the Lamiaceae family which is used in tea and food preparations, cosmetics, and phytomedicals. It has a long tradition as sedative, spasmolytic, hypotensive, and antimicrobial phytopharmaceutical in various cultures [17]. There are to our knowledge so far no indications for the here-described molecular mechanism of PPAR activation and the usage of ethanolic balm extracts as efficient antidiabetics or antihyperlipidemics [18].

Essential oil prepared from the leaves of lemon balm was shown to have general antioxidative properties and consequently reduced plasma glucose and triacylglycerols in monogenic-diabetic $\mathrm{db} / \mathrm{db}$ mice. In addition, balm oil improved dyslipidemia in apolipoprotein E2 transgenic mice through sterol regulatory element-binding protein dependent mechanisms [19]. Aqueous Melissa extracts further showed hepatoprotective effects in hyperlipidemic rats [20].

Due to their property as nutritional sensors nuclear receptors such as the PPARs are interesting targets for dietary intervention. The present study demonstrated that our ethanolic LBE contains ligands for all three PPAR subtypes (Ki values ranging from 0.4 to $1.2 \mu \mathrm{g} / \mathrm{mL}$ ), indicating that the complex extract comprises many active ingredients that may address multiple other targets relevant for the metabolic syndrome. LBEs contain a large amount of phytochemicals, including phenolic acids (rosmarinic acid, coumaric acid, caffeic acid and derivatives, protocatechuic acid, ferulic acid, chlorogenic acid), flavonoids (quercetin, luteolin, apigenin, and their glucosides), sesquiterpenes ( $\beta$-caryophyllene and germacrene), monoterpenes ( $\beta$-pinene), and triterpenes [12, 17, 18, 21]. Many of these small molecules have been identified as PPAR ligands, including rosmarinic acid, apigenin, quercetin, and luteolin [22].

To further characterise the LBE we subfractionated the extract and observed in all ten subfractions PPAR- $\gamma$-binding constituents ( $\mathrm{Ki}=0.3$ to $7.5 \mu \mathrm{g} / \mathrm{mL}$, Supplementary Information Fig. 4A), whereas the Ki for the full LBE was $1.2 \mu \mathrm{g} / \mathrm{mL}$. Noteworthy, the binding affinity correlated well 


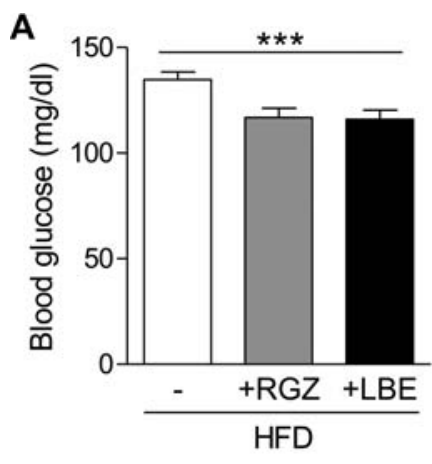

D

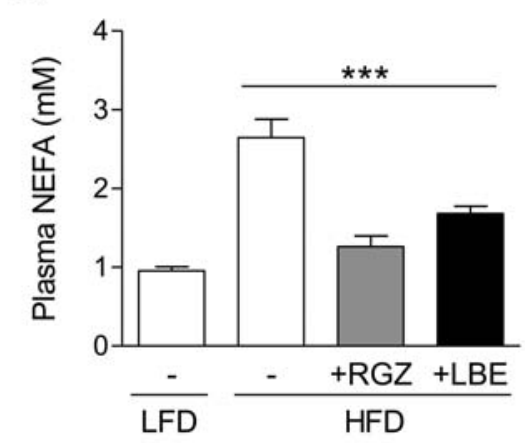

$\mathbf{F}$

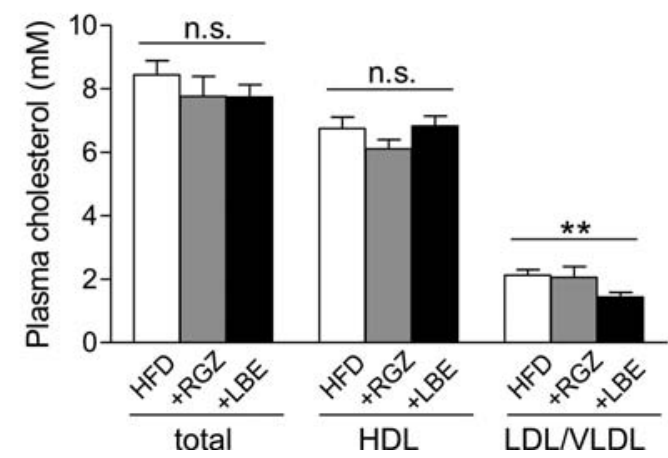

B

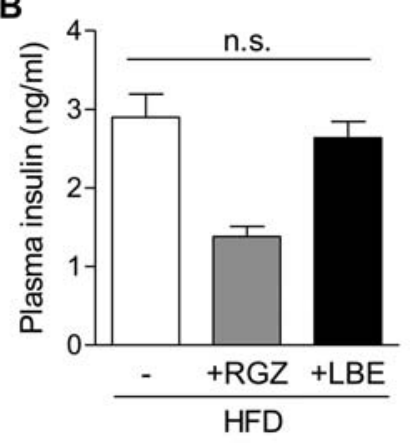

E
C

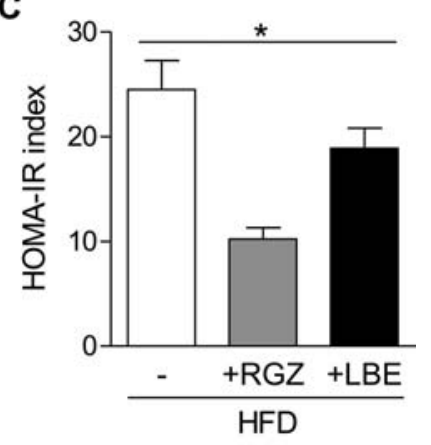

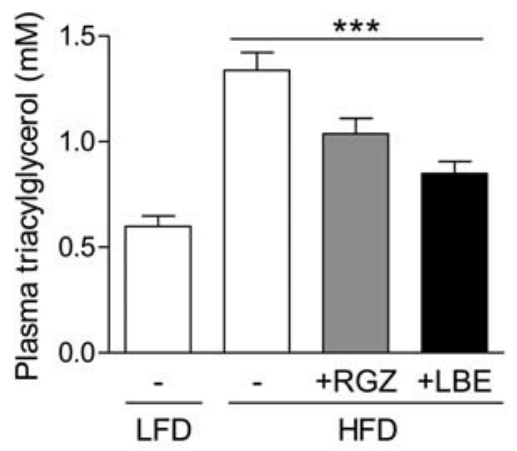

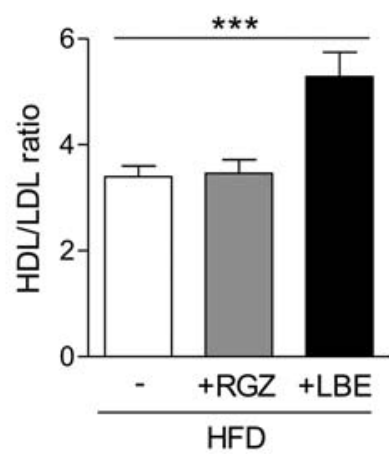

Figure 2. Antidiabetic and antihyperlipidemic effects of LBE in insulin-resistent diet-induced obese mice. (A) Fasting blood glucose of untreated HFD-fed mice or mice treated for 2 weeks with RGZ or LBE. (B) Fasting plasma insulin after 2 weeks of treatment. (C) Effect of treatment for 2 weeks on insulin resistance determined by homeostatis model assessment of insulin resistance (HOMA-IR). (D) Fasting nonesterified fatty acids after 6 weeks of treatment. (E) Fasting plasma triacylglycerol after 6 weeks of treatment. (F) Fasting plasma total, HDL, and LDL/VLDL cholesterol, and HDL/LDL ratio in diet-induced obese mice after 6 weeks of treatment. Data are expressed as mean \pm SEM. ${ }^{*} p \leq 0.05 ;{ }^{*} p \leq 0.01 ;{ }^{* *} p \leq 0.001$, versus vehicle-treated HFD-fed mice. LFD, low-fat diet; HFD, high-fat diet; VEH, vehicle ( $n=$ 13-14); RGZ, rosiglitazone ( $n=8-14)$; LBE, lemon balm extract $(n=13-14)$.

with the hydrophobicity of the subfraction, confirming the well-known preference of PPAR- $\gamma$ for hydrophobic ligands. However, PPAR-controlled gene expression in the adipocytes showed lower activation for the stronger PPAR-binding fractions, suggesting counteracting effects of hydrophobicity of some molecules in the cellular context (Supplementary Information Fig. 4B). These data suggest that optimizing the extract composition for binding affinity or reporter gene effective concentrations would be inefficient for identifiying the antidiabetic and hypolipidemic constituents in the LBE. Note- worthy, transcriptional activation of the subfractions was very diverse for different genes. For example, we observed similar expression of adiponectin in contrast to varying effects on NR1H3. These data indicate complex, in part unpredictable effects on cellular gene regulation due to the so far only partly understood concerted actions of various transcriptional regulators.

Consequently, it seems useless to search for few specific PPAR-modulating ligands or fractions in the whole extract, whereas the synergistic or additive effects of many 
compounds of the LBE such as (poly)phenols may mediate the observed activation of PPARs. These effects may have resulted in the observed improved physiology of insulinresistant obese mice treated with the LBE mixture, which would have been lost, at least in part, by breaking the mixture down to few single compounds.

The holistic phytomedical poly-drug/poly-target concept may add fruitful approaches for developing functional food or nutraceuticals for preventing and treating multifactorial metabolic diseases [23]. Notably, this approach may overcome the mentioned problems with undesirable side effects of strong synthetic PPAR agonists. Moreover, many plant extracts such as the LBE presented here showed good safety profiles in humans.

The reasonable concentration of LBE applied in this study holds promise for application in humans to prevent and counteract polyetiological age-associated metabolic diseases such as type 2 diabetes and related disorders.

We are deeply grateful to K. Hansen, C. Franke, U. Schroeder, and L. Hartmann for technical assistance during the animal studies. Our work is supported by the German Ministry for Education and Research (BMBF, grant number 0315082 (01EA1303)), the European Union (FP7/2007-2013, under grant agreement number $n^{\circ} 262055$ (ESGI)), and the Max Planck Society. This work is part of the Ph.D. thesis of C. Weidner.

Potential conflict of interest statement: H. Abdel-Aziz and O. Kelber are employed by Steigerwald Arzneimittelwerk GmbH, a company that sells phytomedical products. The authors have declared no conflict of interest.

\section{References}

[1] Smyth, S., Heron, A., Diabetes and obesity: the twin epidemics. Nat. Med. 2006, 12, 75-80.

[2] Kliewer, S. A., Xu, H. E., Lambert, M. H., Willson, T. M., Peroxisome proliferator-activated receptors: from genes to physiology. Recent Prog. Horm. Res. 2001, 56, 239-263.

[3] Michalik, L., Auwerx, J., Berger, J. P., Chatterjee, V. K. et al., International Union of Pharmacology. LXI. Peroxisome proliferator-activated receptors. Pharmacol. Rev. 2006, 58, 726-741.

[4] Berger, J., Moller, D. E., The mechanisms of action of PPARs. Annu. Rev. Med. 2002, 53, 409-435.

[5] [Anon], Regulators restrict Avandia in the US and suspend it in the EU. Nat. Rev. Drug Discov. 2010, 9, 828-828.

[6] Pourcet, B., Fruchart, J. C., Staels, B., Glineur, C., Selective PPAR modulators, dual and pan PPAR agonists: multimodal drugs for the treatment of type 2 diabetes and atherosclerosis. Expert Opin. Emerg. Drugs 2006, 11, 379-401.

[7] Bailey, C. J., Learning from tesaglitazar. Diab. Vasc. Dis. Res. 2007, 4, 161-162.

[8] Quideau, S., Deffieux, D., Douat-Casassus, C., Pouysegu, L., Plant polyphenols: chemical properties, biological activ- ities, and synthesis. Angew. Chem. Int. Ed. Engl. 2011, 50, 586-621.

[9] Weidner, C., de Groot, J. C., Prasad, A., Freiwald, A. et al., Amorfrutins are potent antidiabetic dietary natural products. Proc. Natl. Acad. Sci. USA 2012, 109, 7257-7262.

[10] Muller, M., Kersten, S., Nutrigenomics: goals and strategies. Nat. Rev. Genet. 2003, 4, 315-322.

[11] Kroll, U., Cordes, C., Pharmaceutical prerequisites for a multi-target therapy. Phytomedicine 2006, 13, 12-19.

[12] Wegener, T., Wagner, H., The active components and the pharmacological multi-target principle of STW 5 (Iberogast). Phytomedicine 2006, 13, 20-35.

[13] Spiegelman, B. M., Frank, M., Green, H., Molecular cloning of mRNA from 3T3 adipocytes. Regulation of mRNA content for glycerophosphate dehydrogenase and other differentiationdependent proteins during adipocyte development. J. Biol. Chem. 1983, 258, 10083-10089.

[14] Feldmann, R., Fischer, C., Kodelja, V., Behrens, S. et al., Genome-wide analysis of LXRalpha activation reveals new transcriptional networks in human atherosclerotic foam cells. Nucl. Acids Res. 2013, 41, 3518-3531.

[15] Feldmann, R., Geikowski, A., Weidner, C., Witzke, A. et al., Foam cell specific LXRalpha ligand. PLoS One 2013, 8, e57311.

[16] Weidner, C., Wowro, S. J., Freiwald, A., Kawamoto, K. et al., Amorfrutin $B$ is an efficient natural peroxisome proliferatoractivated receptor gamma (PPARgamma) agonist with potent glucose-lowering properties. Diabetologia 2013, 56, 1802-1812.

[17] Ulbricht, C., Brendler, T., Gruenwald, J., Kligler, B. et al., Lemon balm (Melissa officinalis L.): an evidence-based systematic review by the Natural Standard Research Collaboration. J. Herb. Pharmacother. 2005, 5, 71-114.

[18] Chung, M. J., Cho, S. Y., Bhuiyan, M. J., Kim, K. H., Lee, S. J., Anti-diabetic effects of lemon balm (Melissa officinalis) essential oil on glucose- and lipid-regulating enzymes in type 2 diabetic mice. Br. J. Nutr. 2010, 104, 180-188.

[19] Jun, H. J., Lee, J. H., Jia, Y., Hoang, M. H. et al., Melissa officinalis essential oil reduces plasma triglycerides in human apolipoprotein E2 transgenic mice by inhibiting sterol regulatory element-binding protein-1c-dependent fatty acid synthesis. J. Nutr. 2012, 142, 432-440.

[20] Bolkent, S., Yanardag, R., Karabulut-Bulan, O., Yesilyaprak, B., Protective role of Melissa officinalis L. extract on liver of hyperlipidemic rats: a morphological and biochemical study. J. Ethnopharmacol. 2005, 99, 391-398.

[21] Fecka, I., Turek, S., Determination of water-soluble polyphenolic compounds in commercial herbal teas from Lamiaceae: peppermint, melissa, and sage. J. Agric. Food Chem. 2007, 55, 10908-10917.

[22] Mueller, M., Lukas, B., Novak, J., Simoncini, T. et al., Oregano: a source for peroxisome proliferator-activated receptor gamma antagonists. J. Agric. Food Chem. 2008, 56, $11621-11630$.

[23] Wagner, H., Ulrich-Merzenich, G., Synergy research: approaching a new generation of phytopharmaceuticals. Phytomedicine 2009, 16, 97-110. 